\title{
Lateral Pelvic Lymph Node Dissection After Neoadjuvant Chemoradiotherapy in Patients With Rectal Cancer: A Single-Center Experience and Literature Review
}

\author{
Min Chul Kim, Jae Hwan Oh \\ Center for Colorectal Cancer, Research Institute and Hospital, National Cancer Center, Goyang, Korea
}

Purpose: We aimed to evaluate the surgicopathological outcomes of lateral pelvic lymph node dissection (LPLD) and long-term oncological outcomes of selective LPLD after neoadjuvant chemoradiotherapy (nCRT) in patients with locally advanced rectal cancer and compare them to those of total mesorectal excision (TME) alone based on pretreatment magnetic resonance imaging (MRI).

Methods: We compared the TME-alone group (2001-2009, $\mathrm{n}=102)$ with the TME with LPLD group (2011-2016, $\mathrm{n}=69$ ), both groups having lateral lymph nodes (LLNs) of $\geq 5 \mathrm{~mm}$ in short axis diameter. The surgicopathological outcomes were analyzed retrospectively. Oncological outcomes were analyzed using the Kaplan-Meier method.

Results: The rates of overall postoperative 30 -day morbidity ( $42.0 \%$ vs. $26.5 \%, \mathrm{P}=0.095)$ and urinary retention $(13.7 \%$ vs. $10.1 \%, \mathrm{P}=0.484$ ) were not significantly different between the LPLD and TME-alone groups, respectively. Pathologically proven LLN metastasis was identified in $24(34.8 \%)$ LPLD cases after nCRT. The LPLD group showed a lower 5-year local recurrence $(\mathrm{LR})$ rate $(27.9 \%$ vs. $4.6 \%, \mathrm{P}<0.001)$ and better recurrence-free survival $(\mathrm{RFS})(59.6 \%$ vs. $78.2 \%, \mathrm{P}=0.008)$ than those of the TME-alone group, while the 5-year overall survival was not significantly different between the 2 groups (76.2\% vs. $86.5 \%, \mathrm{P}=0.094)$.

Conclusion: This study suggests that LPLD is a safe and feasible procedure. The oncological outcomes suggest that selective LPLD improves LR and RFS in patients with clinically suspicious LLNs on pretreatment MRI. Considering that lateral nodal disease is not common, a multicenter large-scale study is necessary.

Keywords: Rectal cancer; Lateral pelvic lymph node dissection; Neoadjuvant chemoradiotherapy

\section{INTRODUCTION}

The principal role of surgery for locally advanced rectal cancer is to prevent locoregional recurrence. Thus, procedures should be able to remove not only the primary tumor but also its lymphatic system. Gerota in 1895 and Villemin in 1925 demonstrated that

Received: Oct 15, 2021 - Revised: Nov 17, 2021 - Accepted: Nov 24, 2021 Correspondence to: Jae Hwan Oh, M.D., Ph.D.

Center for Colorectal Cancer, Research Institute and Hospital, National

Cancer Center, 323 Ilsan-ro, Ilsandong-gu, Goyang 10408, Korea

Tel: +82-31-920-1515, Fax: +82-31-920-1511

E-mail: jayoh@ncc.re.kr

ORCID: https://orcid.org/0000-0002-5883-5815

(C) 2021 The Korean Society of Coloproctology

This is an open-access article distributed under the terms of the Creative Commons Attribution NonCommercial License (https://creativecommons.org/licenses/by-nc/4.0) which permits unrestricted noncommercial use, distribution, and reproduction in any medium, provided the original work is properly cited. the lower rectum has a distinct lateral lymphatic flow to the iliac nodes. Lateral lymph node (LLN) metastasis has been reported to occur in $15 \%$ to $20 \%$ of patients with locally advanced mid- to low-rectal cancer [1]. However, the management of lateral pelvic nodal disease is oncologically and technically challenging.

In the 1950s, Western surgeons conducted studies regarding extended lymphadenectomy with small case series. It was concluded that improvements in the oncological outcomes were minimal compared to the increased risk of postoperative complications. Although Enker et al. [2] reported a survival advantage when performing lateral pelvic lymph node dissection (LPLD) for Dukes' $\mathrm{C}$ tumors, the technique was gradually discontinued for various reasons including low incidence of lateral nodal disease, high morbidity, and minimal oncological benefit. As extended surgery may not yield good results significantly, this procedure has not been routinely performed in the Western World [2-4]. 
In Japan, Senba conducted an anatomic study on the lymphatics of the rectum in 1927 that showed that the lymphatic vessels were distributed around the internal iliac artery and obturator space. Based on Senba's study, Kuru [5] reported the first clinical study of LPLD in 1940. Since then, LPLD was developed and improved by many Japanese surgeons, mainly those working in the National Cancer Center and Cancer Research Institute Hospital in Japan. As a result, the current Japanese guidelines recommend that for rectal cancer below the peritoneal reflection and invades beyond the muscularis propria, LPLD is to be performed $[6,7]$. In contrast, Dutch and German trials have reported better locoregional control rates in the neoadjuvant (chemo)radiotherapy $(\mathrm{n}(\mathrm{C}) \mathrm{RT}$ ) group, even in patients who underwent total mesorectal excision (TME) alone. Based on these studies, $\mathrm{n}(\mathrm{C}) \mathrm{RT}$ followed by TME is regarded as the standard treatment for locally advanced rectal cancer not only in Western countries but also in South Korea [811]. Most Western surgeons consider LLN metastasis as a systemic disease. In their meta-analysis, Georgiou et al. [12] reported that LPLD was associated with urinary and sexual dysfunction without added oncological benefit, although this study had many limitations. Recently, Emile et al. [13] also conducted a metaanalysis comparing the outcomes of TME with LPLD to TME alone for rectal cancer. Similarly, a majority of studies were from Japanese centers (23 of 29); therefore, most of the patients involved in this study did not undergo neoadjuvant chemoradiotherapy (nCRT), which was different from Western guidelines. Most of studies were retrospective (22 of 29), and patients in 7 studies were treated before the year 2000. In addition, the metaanalysis only included 2 randomized controlled trials (RCT) for the evaluation of LPLD; one is the Japan Clinical Oncology Group (JCOG) 0212 trial, and the other is a small RCT that analyzed patient outcomes after nCRT. In these studies, LPLD did not show any oncological benefit and was associated with an increased complication rate and operation time [13]. LPLD was performed in patients with high-risk disease (enlarged LLN). Comparing the LPLD group with the TME-alone group is the same as comparing the high-risk group with the low-risk group. Therefore, if the patients who underwent LPLD showed similar oncological outcomes to those who did not, the benefit of LPLD could be suggested, as Kong and Chang [14] have pointed out.

The JCOG0212 trial is the only phase 3 RCT comparing TME with LPLD (control arm) and TME alone (experimental arm). This study included pathologically proven adenocarcinoma of the rectum below the peritoneal reflection with clinical stage 2 or 3 and excluded patients with extramesorectal lymph nodes greater than $10 \mathrm{~mm}$ in diameter. None of the patients underwent neoadjuvant chemotherapy or radiotherapy (RT). The primary endpoint of the study was the 5-year recurrence-free survival (RFS) with a noninferiority trial setting. The 5-year RFS in the TME with LPLD and TME-alone groups were $73.4 \%$ and $73.3 \%$, respectively. However, this study could not prove that TME-alone was noninferior to TME with LPLD. Local recurrence (LR) was observed in 44 (12.6\%) and 26 patients (7.4\%) in the TME-alone and TME with LPLD groups, respectively $(\mathrm{P}=0.024)$, while the morbidity and functional outcomes were not different [15-17].

A head-to-head comparison of treatment results for patients with mid- or low-rectal cancer between Japanese and Western treatment strategies might be difficult to accomplish for various technical and ethical reasons. One comparative study, which used data from the Dutch TME trial and National Cancer Center Japan, showed similar 5-year LR rates of $6.9 \%$ in the Japanese extended surgery group and $5.8 \%$ in the Dutch preoperative RT with TME (RT + TME) group; however, the Dutch nonirradiated TME group showed a significantly increased 5-year LR rate (12.1\%). Unexpectedly, the lateral LR rate in the Dutch nonirradiated TME group was only $2.7 \%$ and comprised $24 \%$ of all LRs, which was similar to that of the Japanese group even after LPLD (2.2\%, 35\% of all LR). Presacral recurrences occurred in $3.7 \%$ of the Dutch RT + TME group, $3.2 \%$ of the Dutch nonirradiated TME group, and $0.6 \%$ of the Japanese group. The lateral LR rate in the RT + TME group ( $0.8 \%)$ was significantly lower than that of the nonirradiated TME group (2.7\%), which suggests that RT plays a significant role in the reduction of lateral pelvic recurrence; however, RT could not prevent presacral recurrence. As a result, both extended surgery and preoperative RT with standard TME resulted in better local control in the treatment of distal rectal cancer compared with TME alone. Given the morbidity associated with LPLD, n(C)RT with standard TME surgery was preferred by Western surgeons [18]. Similarly, a study revealed that there was no survival difference between LPLD (in Japan) and TME with postoperative chemoradiotherapy (CRT) in Korea [19]. Meanwhile, nCRT and TME are considered the standard treatments for locally advanced rectal cancer in Western countries and South Korea. Even after nCRT, many LRs were noted in the lateral pelvic wall, which was difficult to remove surgically and caused several problems, including severe pelvic and leg pain and eventually death. Before the adoption of LPLD, our institution reported that most of the LRs after nCRT followed by TME are from LLNs other than those involved in the circumferential resection margins, and that the recurrence rate was related to the size of the LLN [20]. A subsequent study comprised of 900 patients who underwent either preoperative or postoperative CRT showed similar results with our previous report $[20,21]$. Locoregional recurrence developed in 65 patients (7.2\%); 42 (64.6\%) had LLN recurrence, 20 of which $(47.6 \%)$ had no distant metastasis. The 5-year LLN RFS rates of patients with LLN short axis diameters (SADs) measuring $<5 \mathrm{~mm}, 5$ to $<10 \mathrm{~mm}$, and $\geq 10 \mathrm{~mm}$ were $98.2 \%, 91.7 \%$, and $40.1 \%$, respectively $(\mathrm{P}<0.05)$. This suggests that the LLN size was an important risk factor for lateral pelvic recurrence [21].

Another study was conducted with patients who had available data regarding both pretreatment and restaging magnetic resonance imaging (MRI). Of the 580 patients, 157 patients $(27.1 \%)$ presented with LLNs with SADs of $\geq 5 \mathrm{~mm}$ on pretreatment MRI (clinically suspicious LLN). In the multivariate analysis, the LLN 
response to nCRT was significantly associated with the overall survival (OS), LLN RFS, and locoregional RFS. Of 59 patients who had LLNs with SADs of $\geq 5 \mathrm{~mm}$ at restaging MRI, 15 patients (25.4\%) showed LLN recurrence. On the other hand, of 98 patients who had LLNs with SADs of $<5 \mathrm{~mm}$ at restaging MRI, 8 patients (8.2\%) showed LLN recurrence [22].

Majority of Western and Korean surgeons have regarded lateral nodal disease as systemic metastasis, and patients with this cannot be treated with LPLD. However, they have recognized that nCRT alone might not be sufficient to prevent LLN recurrence. In addition, approximately $40 \%$ of patients with LR had no distant metastasis, suggesting that LLN metastasis is not systemic and could possibly benefit from LPLD [23]. Meanwhile, Japanese surgeons are adopting nCRT with selective LPLD to prevent LR and avoid overtreatment [24].

An international multicenter pooled analysis revealed that LPLD could significantly contribute to the control of lateral LR in selected patients [25]. On the pretreatment MRI of 1,216 patients, 192 (15.8\%) had LLNs with SADs of $\geq 7 \mathrm{~mm}$. LR developed in 108 patients (5-year LR rate, $10.0 \%$ ), of which 59 (54.6\%) were LLN recurrence (5-year LLN recurrence rate, 5.5\%).

We recognized that LLN recurrence was a major cause of LR (more than half of LR) even after nCRT, which was related to the LLN size and CRT response based on restaging MRI. However, because lateral nodal disease is uncommon and Japanese surgeons have adopted nCRT to treat locally advanced mid- or lowrectal cancer recently, there are limited data on the role of LPLD after nCRT.

We have already published oncological outcomes of TME with LPLD comparing TME alone after nCRT [26]. The present study aimed to update our oncological data after the extension of the follow-up period and compare the surgicopathological outcomes between the LPLD group and TME-alone group.

\section{METHODS}

\section{Study design}

This study is an update of a previously published retrospective single-center study that included patients with rectal cancer treated with nCRT since 2001 at the National Cancer Center, Goyang, Republic of Korea. The study was approved by the Institutional Review Board of the National Cancer Center (No. NCC2021-0261). An informed consent was not required because of the study design being retrospective.

\section{Study populations}

The inclusion and exclusion criteria and patients and treatment characteristics have been previously reported [26]. Briefly, between 2001 and 2009, patients with locally advanced rectal cancer (cT3 or cT4) with enlarged LLNs were treated with preoperative long-course CRT followed by TME without LPLD. However, we selectively performed LPLD to patients with LLNs with SADs of $\geq 5 \mathrm{~mm}$ on pretreatment MRI since mid-2010. We set a cutoff value of LLNs with SADs of $\geq 5 \mathrm{~mm}$ on pretreatment MRI for LPLD based on our previous study using the receiver operating characteristic (ROC) curve.

We included patients treated in 2001 to 2009 (period I; TMEalone group) and 2011 to 2016 (period II; LPLD group). We excluded patients treated in 2010 to minimize the learning curve effect and selection bias.

Staging workups were performed in all patients before nCRT, which included digital rectal examination, complete blood count, liver function tests, carcinoembryonic antigen (CEA) level, video colonoscopy, chest radiography, computed tomography (CT) of the abdomen and chest, and rectal MRI using the protocols described in our previous report [26]. Restaging rectal MRI was performed to evaluate the tumor response to CRT within 1 week prior to surgery using the same protocol as the initial MRI.

Our institutional policy defined clinically suspected LLNs as those with SADs of $\geq 5 \mathrm{~mm}$ on pretreatment MRI. There were 2 groups for clinically suspected LLNs: responsive LLNs, whose SADs were $<5 \mathrm{~mm}$ on restaging MRI after nCRT; persistent LLNs, whose SADs were $\geq 5 \mathrm{~mm}$ on restaging MRI.

\section{Treatment \\ Radiotherapy}

Preoperative RT delivered 45 Gy in 25 fractions to the entire pelvis, followed by a 5.4-Gy boost in 3 fractions to the primary tumor. All patients underwent CT simulation for 3-dimensional conformal RT planning, and a 3-field treatment plan was used with a 6-MV posterior-anterior photon field and 15-MV opposed lateral photon beams. The radiation field volume enclosed the rectal tumor, mesorectum, presacral space, entire sacral hollow, and regional lymphatics, including the perirectal, internal iliac, presacral, and distal common iliac nodes.

\section{Chemotherapy}

Preoperative concurrent chemotherapy via a 5 -fluorouracil (5-FU)-based regimen was initiated on the first day of pelvic RT. No patients in this study received induction or consolidation chemotherapy. After surgery, all patients were considered for adjuvant chemotherapy regardless of their pathological stage.

\section{Surgery}

TME was performed 6 to 8 weeks after the completion of nCRT. LPLD was performed by multiple surgeons in our institution following either an open or laparoscopic approach with autonomic nerve preservation.

\section{Lateral pelvic lymph node dissection}

Lateral dissection was performed to excise LNs along the internal iliac and middle rectal vessels and in the obturator space. In the absence of clinically suspicious LNs, LN dissection was not usually performed along the common iliac artery [27]. The lateral, 
medial, cranial, caudal, and dorsal borders of the LPLD are the external iliac artery, pelvic plexus, bifurcation of the common iliac artery, levator ani muscle, and sciatic nerve, respectively [24].

\section{Follow-up and evaluation}

All patients underwent standardized follow-up, including a physical examination, complete blood count, serum CEA test, and liver function tests, every 3 months for the first 2 years and every 6 months thereafter, as well as chest and abdominopelvic CT scan every 6 months. Colonoscopy was performed 1 year postoperatively and then once every 2 years. Recurrence was proven by surgical resection, biopsy or cytology, and/or radiological findings, which increased in size over time: LR was defined as tumor recurrence within the pelvic cavity, and distant (metastatic) recurrence (DR) was defined as any recurrence outside of the pelvic cavity. LLN recurrence was defined as recurrence in the LLN-bearing areas outside the mesorectal fascia and along the obturator, internal iliac, and external iliac vessels.

\section{Statistical analysis}

We defined LR, DR, cancer-specific survival (CSS), and OS as the time from surgery to the occurrence of LR, DR, death caused by rectal cancer, and death by any cause, respectively. Similarly, RFS was defined as the time from surgery to any LR, distant metastasis, or death. To balance the follow-up times, the end of the study was set at February 28, 2011, for groups treated between 2001 and 2009 and October 31, 2020, for groups treated between 2011 and 2016.

Continuous variables are presented as means \pm standard deviations or medians (interquartile ranges [IQRs]), according to the normality of distributions, while categorical variables are presented as frequencies (percentages). Continuous and categorical variables were compared using the Student t-test or the MannWhitney U-test and the chi-square test or Fisher exact test, respectively. The Kaplan-Meier method was used to estimate the survival rates and visualize the survival curves during follow-up. Subsequently, the log-rank test was performed to compare the survival curves among the groups. All results with 2-tailed P-values of $<0.05$ were considered statistically significant.

All statistical analyses were performed using SAS software (version 9.4; SAS Inc., Cary, NC, USA) and R project software (version 3.6.2; http://www.R-project.org).

\section{RESULTS}

In this study, 918 patients were analyzed after the exclusion of 23 patients with distant metastasis; among these, 171 patients (18.6\%) had clinically suspicious LLNs. Also, 102 of 576 patients (17.7\%) underwent TME alone (2001-2009) whereas 69 of 342 patients (20.2\%) underwent TME with LPLD (2011-2016).

The baseline characteristics of the patients, tumor, and treatment modality are summarized in Table 1 . There was no signifi-
Table 1. Demographics of patients with lateral lymph node of $\geq 5$ $\mathrm{mm}$ in the short axis on pretreatment rectal magnetic resonance imaging

\begin{tabular}{|c|c|c|c|}
\hline Variable & $\begin{array}{l}\text { TME alone } \\
(n=102)\end{array}$ & $\begin{array}{l}\text { TME with } \\
\text { LPLD } \\
(n=69)\end{array}$ & P-value \\
\hline No. of patients & 102 & 69 & \\
\hline Sex & & & 0.052 \\
\hline Female & $31(30.4)$ & $31(44.9)$ & \\
\hline Male & $71(69.6)$ & $38(55.1)$ & \\
\hline Age (yr) & $55.2 \pm 10.8$ & $57.9 \pm 12.1$ & 0.137 \\
\hline Tumor location from the anal verge $(\mathrm{cm})$ & & & 0.960 \\
\hline$>5$ & $41(40.2)$ & $28(40.6)$ & \\
\hline$\leq 5$ & $61(59.8)$ & $41(59.4)$ & \\
\hline Pre-CRT CEA level (ng/mL) & & & 0.146 \\
\hline$\leq 5$ & $36(35.3)$ & $32(46.4)$ & \\
\hline$>5$ & $66(64.7)$ & 37 (53.6) & \\
\hline Post-CRT CEA level (ng/mL) & & & 0.050 \\
\hline$\leq 5$ & $11(10.8)$ & $15(21.7)$ & \\
\hline$>5$ & $91(89.2)$ & $54(78.3)$ & \\
\hline Preoperative concurrent chemotherapy & & & $<0.001$ \\
\hline 5-FU and leucovorin & $63(61.8)$ & $19(27.5)$ & \\
\hline Capecitabine & $18(17.6)$ & $50(72.5)$ & \\
\hline Capecitabine + irinotecan & $15(14.7)$ & $0(0.0)$ & \\
\hline Cetuximab & $6(5.9)$ & $0(0.0)$ & \\
\hline Adjuvant chemotherapy & & & $<0.001$ \\
\hline No & $2(2.0)$ & $11(15.9)$ & \\
\hline 5-FU or capecitabine & 94 (92.2) & $32(46.4)$ & \\
\hline FOLFOX/XELOX/FOLFIRI & $6(5.9)$ & $26(37.7)$ & \\
\hline cT stage & & & 0.004 \\
\hline 2 & $0(0)$ & $1(1.4)$ & \\
\hline 3 & $96(94.1)$ & 54 (78.3) & \\
\hline 4 & $6(5.9)$ & $14(20.3)$ & \\
\hline cN stage & & & $<0.001$ \\
\hline 0 & $3(2.9)$ & $0(0)$ & \\
\hline 1 & $69(67.6)$ & $69(100)$ & \\
\hline 2 & $30(29.4)$ & $0(0)$ & \\
\hline Radiotherapy response & & & 0.004 \\
\hline Persistent & $35(34.3)$ & $39(56.5)$ & \\
\hline Responsive & $67(65.7)$ & $30(43.5)$ & \\
\hline
\end{tabular}

Values are presented as number only, number (\%), or mean \pm standard deviation. TME, total mesorectal excision; LPLD, lateral pelvic lymph node dissection; CEA, carcinoembryonic antigen; CRT, chemoradiotherapy; 5-FU, 5-fluorouracil; FOLFOX, leucovorin, 5-FU, and oxaliplatin; XELOX, capecitabine and oxaliplatin; FOLFIRI, leucovorin, 5-FU, and irinotecan. 


\section{Coloproctology $\begin{array}{r}\text { Annals of } \\ \text { Cancer: A Single-Center Experie }\end{array}$}

cant difference in sex, age, tumor location, and CEA level between the groups. In the LPLD group, the proportion of patients with clinical stage T4 was increased, and all were classified as clinical stage N1. As a preoperative concurrent chemotherapeutic agent,
5-FU was mainly used in the TME-alone group whereas capecitabine was dominantly used in the LPLD group. In the TME with LPLD group, more patients received adjuvant chemotherapy, and significantly more patients had persistent LN to

Table 2. Surgicopathological outcomes of LLN dissection

\begin{tabular}{|c|c|c|c|}
\hline Variable & TME alone $(n=102)$ & TME with LPLD $(n=69)$ & P-value \\
\hline Operation type & & & $<0.001$ \\
\hline Laparoscopic & $26(25.5)$ & $58(84.1)$ & \\
\hline Open & $76(74.5)$ & $11(15.9)$ & \\
\hline Operation & & & $<0.001$ \\
\hline Sphincter-preserving operation & $81(79.4)$ & $67(97.1)$ & \\
\hline Abdominoperineal resection & $21(20.6)$ & $2(2.9)$ & \\
\hline Operative time (min) & $220.0(177.0-280.0)$ & $330.0(270.0-425.0)$ & $<0.001$ \\
\hline Transfusion & & & 0.442 \\
\hline No & 99 (97.1) & $65(94.2)$ & \\
\hline Yes & $3(2.9)$ & $4(5.8)$ & \\
\hline Estimated blood loss (mL) & $200.0(150.0-350.0)$ & $100.0(50.0-200.0)$ & $<0.001$ \\
\hline Length of hospital stay (day) & $10.0(9.0-14.0)$ & $11.0(9.0-15.0)$ & 0.567 \\
\hline Urinary retention & & & 0.484 \\
\hline No & $88(86.3)$ & $62(89.9)$ & \\
\hline Yes & $14(13.7)$ & $7(10.1)$ & \\
\hline Morbidity (postoperative 30 days) & & & 0.095 \\
\hline No & $75(73.5)$ & $40(58.0)$ & \\
\hline CD I, II & $23(22.5)$ & $25(36.2)$ & \\
\hline CD III, IV & $4(3.9)$ & $4(5.8)$ & \\
\hline Tumor size $(\mathrm{cm})$ & $5.0(4.0-6.0)$ & $5.0(4.0-5.0)$ & 0.004 \\
\hline Histologic grade & & & 0.001 \\
\hline Well & $4(3.9)$ & $15(21.7)$ & \\
\hline Moderate & $86(84.3)$ & $49(71.0)$ & \\
\hline Poor/mucinous/signet ring cell & $12(11.8)$ & $5(7.2)$ & \\
\hline ypT stage & & & 0.226 \\
\hline T0-1 & 22 (21.6) & $8(11.6)$ & \\
\hline $\mathrm{T} 2$ & $21(20.6)$ & $13(18.8)$ & \\
\hline T3 & $55(53.9)$ & $42(60.9)$ & \\
\hline T4 & $4(3.9)$ & $6(8.7)$ & \\
\hline ypN stage & & & 0.14 \\
\hline NO & $64(62.7)$ & $33(47.8)$ & \\
\hline N1 & $23(22.5)$ & $20(29.0)$ & \\
\hline N2 & $15(14.7)$ & $16(23.2)$ & \\
\hline Tumor regression grade & & & 0.084 \\
\hline Dworak grade 0-2 & $76(74.5)$ & $59(85.5)$ & \\
\hline Dworak grade 3-4 & $26(25.5)$ & $10(14.5)$ & \\
\hline
\end{tabular}


Table 2. Continued

\begin{tabular}{|c|c|c|c|}
\hline Variable & TME alone $(n=102)$ & TME with LPLD $(n=69)$ & P-value \\
\hline Radial resection margin & & & 0.128 \\
\hline Negative & 90 (88.2) & $55(79.7)$ & \\
\hline Positive & $12(11.8)$ & $14(20.3)$ & \\
\hline Ro & & & 0.302 \\
\hline RO & $96(94.1)$ & $62(89.9)$ & \\
\hline $\mathrm{R} 1$ & $6(5.9)$ & $7(10.1)$ & \\
\hline \multicolumn{4}{|l|}{ Pathological LLN } \\
\hline No metastasis & & $45(65.2)$ & \\
\hline Metastasis & & $24(34.8)$ & \\
\hline$<5 \mathrm{~mm}$ on restaging $\mathrm{MRl}$ & & $4(16.7)$ & \\
\hline$\geq 5 \mathrm{~mm}$ on restaging $\mathrm{MRl}$ & & 20 (83.3) & \\
\hline
\end{tabular}

Values are presented as number (\%) or median (interquartile range).

TME, total mesorectal excision; LPLD, lateral pelvic lymph node dissection; CD, Clavien-Dindo classification; LLN, lateral lymph node; MRI, magnetic resonance imaging.

nCRT than those of the TME-alone group (Table 1).

Table 2 shows the results of the surgicopathological outcomes between the 2 groups. The rates of laparoscopic procedures and sphincter-preserving operations were significantly higher in the LPLD group. In the TME with LPLD group, the operation times were longer, but the estimated blood loss was significantly less. Between the 2 groups, there were no significant differences in the transfusion rates during the perioperative period and length of hospital stay or complications, such as urinary retention. Unlike the clinical stage, there was no significant difference in the pathological stage, and there was no difference between the 2 groups in terms of the rates of circumferential resection margin positive and R0. Of the 69 patients who underwent LPLD, 30 (43.5\%) showed responsive LLNs and 39 (56.5\%) showed persistent LLNs. In addition, pathologically proven LLN metastasis was identified in 24 of 69 patients (34.8\%). Of these patients, 4 of 30 (13.3\%) had responsive LLNs and 20 of 39 (51.3\%) had persistent LLNs.

Fig. 1 shows the Kaplan-Meier curve comparing the LR, DR, RFS, CSS, and OS of the 2 groups. The median follow-up periods were 72.9 and 73.0 months for the TME-alone and TME with LPLD groups, respectively. The 5-year LR rates were $27.9 \%$ and $4.6 \%$ for the TME-alone and TME with LPLD groups, respectively $(\mathrm{P}<0.001)$ (Fig. 1A). The 5-year DR rates for the TMEalone and TME with LPLD groups were $34.1 \%$ and $15.0 \%$, respectively $(\mathrm{P}=0.005)$ (Fig. $1 \mathrm{~B})$. The 5-year RFS rates were confirmed to be $59.6 \%$ and $78.2 \%$ for the TME-alone and TME with LPLD groups, respectively $(\mathrm{P}=0.008)$ (Fig. 1C). The 5-year CSS rate was $78.7 \%$ and $89.3 \%$ for the TME-alone and TME with LPLD groups, respectively $(\mathrm{P}=0.068)(\mathrm{Fig}$. 1D). The 5-year OS rate was $76.2 \%$ and $86.5 \%$ for the TME-alone and TME with LPLD groups, respectively $(\mathrm{P}=0.094)$ (Fig. 1E). This trend was also observed in the subgroup analysis for patients with enlarged lateral pelvic LNs of $\geq 5 \mathrm{~mm}$ after nCRT (Supplementary Fig. 1).
The LPLD group showed a lower 5-year LR (46.1\% vs. $5.5 \%$, $\mathrm{P}<0.001)$, 5-year DR (47.9\% vs. $16.6 \%, \mathrm{P}=0.003)$, and improved 5 -year RFS ( $42.7 \%$ vs. $71.7 \%, \mathrm{P}=0.009)$. The 5 -year CSS $(67.0 \%$ vs. $86.5 \%, \mathrm{P}=0.051)$ and 5 -year OS rates $(67.0 \%$ vs. $86.5 \%$, $\mathrm{P}=0.166$ ) were not significantly different between the 2 groups. However, in patients whose lateral pelvic $\mathrm{LN}$ size decreased to $<5$ $\mathrm{mm}$ after preoperative CRT, a statistically significant difference was found only in the 5 -year LR ( $17.6 \%$ vs. $3.3 \%, \mathrm{P}=0.025)$ and RFS (68.4\% vs. $86.7 \%, \mathrm{P}=0.034$ ) of the LPLD group (Supplementary Fig. 2).

The pattern of LR was different, according to the LPLD status (Table 3). The follow-up period of the TME with LPLD group was extended by 3 years compared with the previous study, but no additional LR or DR was identified. In the TME-alone group, 19 of 25 patients (76.0\%) had LR in the lateral pelvis, while 6 of 25 (24.0\%) had central LR that was in the anastomosis or presacral area. In contrast, all 3 LRs in the TME with LPLD group were centrally located. DR was also significantly less in the TME with LPLD group ( $32.4 \%$ vs. $14.5 \%, \mathrm{P}=0.014)$. The lungs were the main initial DR site in the TME-alone group, but this was not evident in the TME with LPLD group.

\section{DISCUSSION}

In this study of 918 patients, $171(18.6 \%)$ had clinically suspicious LLNs (SAD $\geq 5 \mathrm{~mm}$ ) before nCRT. This study showed that although the operation time was longer in the LPLD group, the rate of morbidity and urinary retention was similar between the LPLD and TME-alone groups. Pathologically proven LLN metastasis was identified in 24 of 69 patients (34.8\%) after nCRT. The extension of the follow-up period of the TME with LPLD group did not change the previously reported oncological outcomes. Selective LPLD in patients with clinically suspicious LLNs was associ- 


\section{$\begin{array}{rrr}\text { Coloproctology } & \text { Cancer: A Single-Center Experien } \\ \text { Chul Kim and Jae Hwan Oh }\end{array}$}
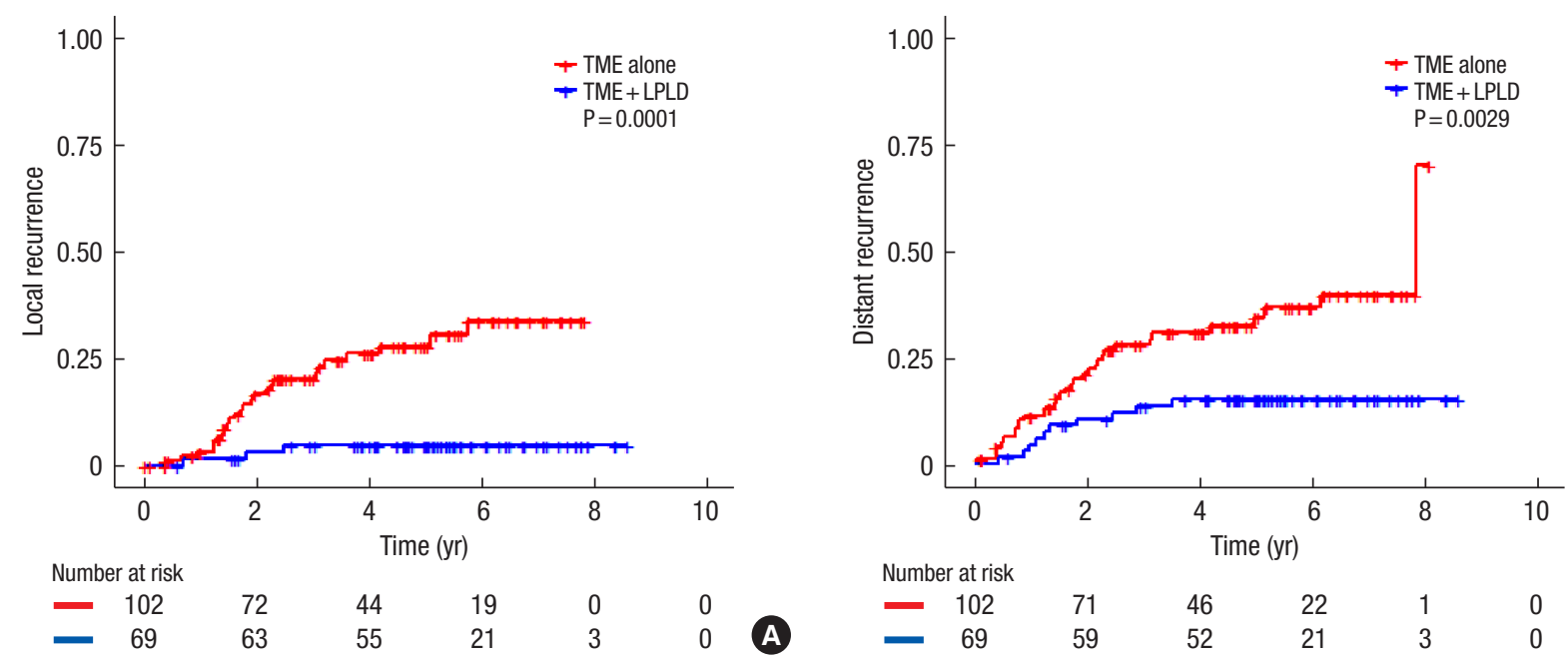

B
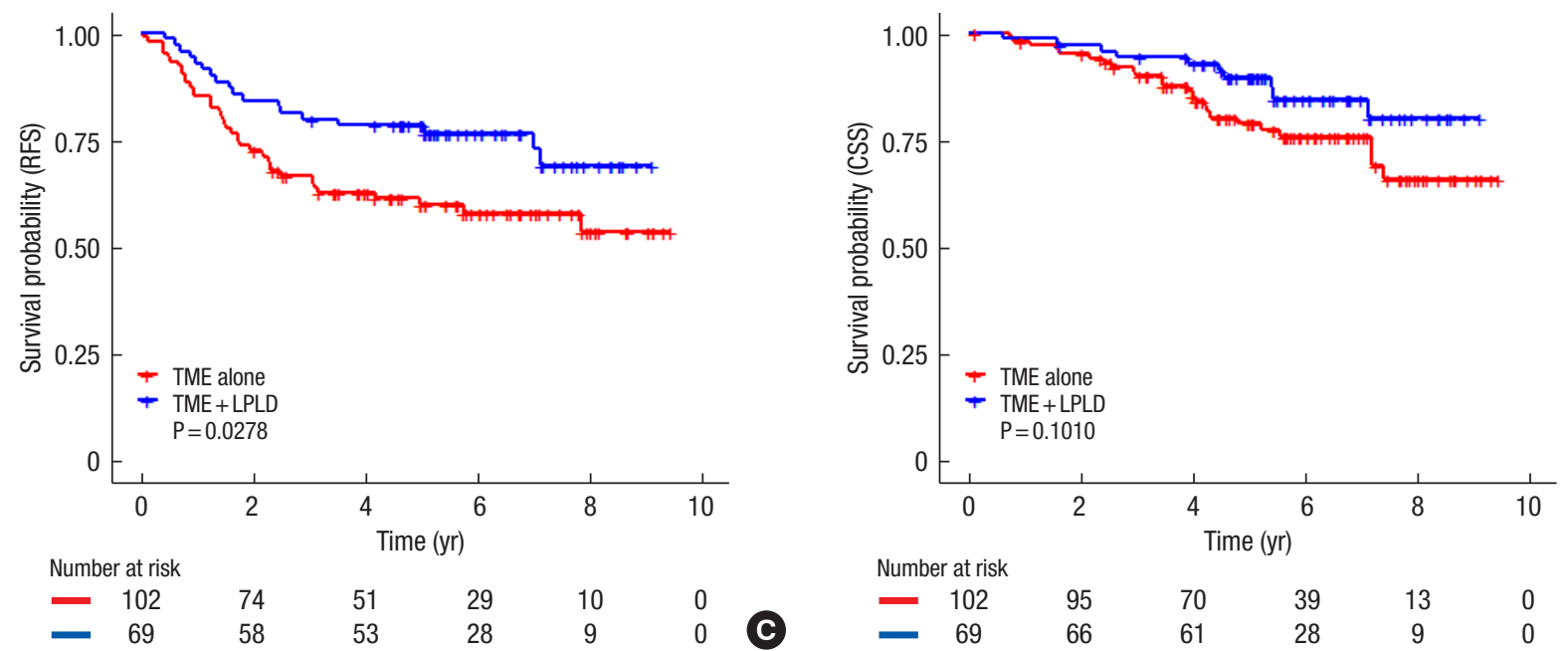

$\begin{array}{ccccccc} & \text { Number at risk } & & & & & \\ \text { C } & 102 & 95 & 70 & 39 & 13 & 0 \\ & 69 & 66 & 61 & 28 & 9 & 0\end{array}$

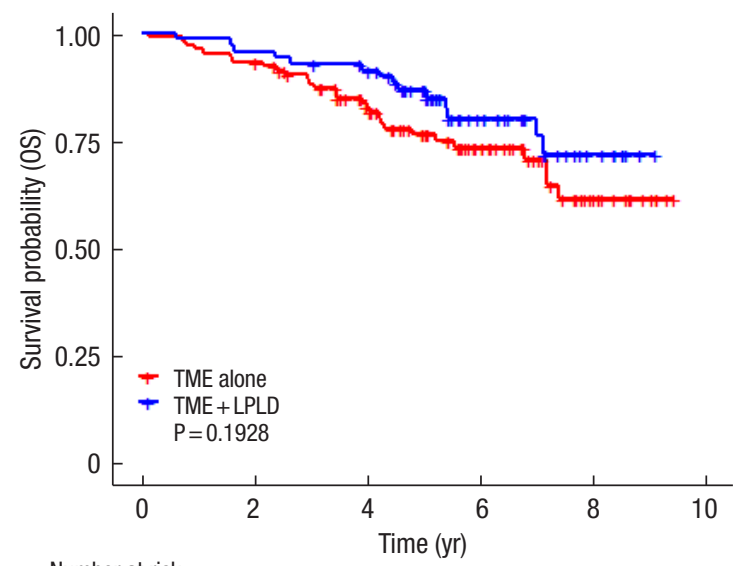

$\begin{array}{cccccc}\text { Number at risk } & & & & & \\ 102 & 95 & 70 & 39 & 13 & 0 \\ 69 & 66 & 61 & 28 & 9 & 0\end{array}$

Fig. 1. Kaplan-Meier curves of (A) cumulative incidence of local recurrence, (B) cumulative incidence of distant recurrence, (C) recurrencefree survival (RFS), (D) cancer-specific survival (CSS), and (E) overall survival (OS) according to patient groups (total mesorectal excision [TME]-alone group vs. TME with lateral pelvic lymph node dissection [LPLD] group). 
Table 3. Pattern of recurrence

\begin{tabular}{lccc}
\hline Variable & $\begin{array}{c}\text { TME alone } \\
(\mathrm{n}=102)\end{array}$ & $\begin{array}{c}\text { TME with LPLD } \\
(\mathrm{n}=69)\end{array}$ & P-value \\
\hline Local recurrence & $25(24.5)$ & $3(4.3)$ & 0.001 \\
$\begin{array}{l}\text { Location of local recurrence } \\
\quad\end{array}$ & $6(5.9)$ & $3(4.3)$ & 0.026 \\
$\quad$ Central & $19(18.6)$ & $0(0)$ & \\
Lateral pelvis & $33(32.4)$ & $10(14.5)$ & 0.014 \\
Distant recurrence & & & 0.049 \\
Location of initial distant recurrence & $21(20.6)$ & $3(4.3)$ & \\
Lung & $4(3.9)$ & $0(0)$ & \\
Liver & $5(4.9)$ & $4(5.8)$ & \\
Inguinal LN or PAN & $3(2.9)$ & $3(4.3)$ & \\
Others & &
\end{tabular}

Values are presented as number (\%).

TME, total mesorectal excision; LPLD, lateral pelvic lymph node dissection; LN, lymph node; PAN, paraaortic LN.

ated with a lower LR rate and better RFS; however, the OS was not significantly improved after selective LPLD. Notably, lateral recurrence was not evident after LPLD. These findings suggest that LPLD plays a major role in the prevention of lateral LR.

\section{What is the cutoff value for "clinically suspicious" lateral lymph nodes?}

The Japanese indication for LPLD is based on the possibility of LLN metastasis of the primary tumor, such as Rb (low margin of tumor located below the peritoneal reflection) and clinical stage T3 or T4, irrespective of LLN size. Unlike in Western countries, $\mathrm{n}(\mathrm{C}) \mathrm{RT}$ is not a preferred treatment method for patients with locally advanced rectal cancer in Japan [6]. On the other hand, the National Comprehensive Cancer Network guidelines recommend nCRT in patients with locally advanced rectal cancer but not an extended lymphadenectomy unless LLNs are clinically suspicious for malignancy; however, the definition of "clinically suspicious" is not clear [28].

Although MRI has become a standard imaging modality for the preoperative evaluation of rectal cancer, its diagnostic accuracy for the nodal status is still less reliable than that of local tumor staging [29]. The size of the LLN before treatment has been known as the main factor for predicting lateral pelvic recurrences and metastasis to LNs, even without a consensus regarding the size criteria. In addition to size, the margin status and signal intensity of LNs plays an important role in predicting its metastatic characteristics [30]. However, because of changes in the morphological characteristics after (C)RT, it is difficult to determine whether LN metastasis is present. A decreased LLN size after nCRT also makes the evaluation of these morphological features difficult. Thus, the size criteria may be a more reliable indicator [31].

Ogawa et al. $[32,33]$ reported that LLN metastases could simply and effectively be evaluated based on the size of LNs, using a cut- off SAD value of $\geq 5 \mathrm{~mm}$ on $\mathrm{MRI}$, which was an important predictor of LLN metastasis in a multi-institutional study conducted by the LN committee of the Japanese Society for Cancer of the Colon and Rectum. However, Ogura et al. [34] reported that the malignant features of LLN with SAD of $\geq 4 \mathrm{~mm}$ on restaging MRI were also a reliable predictor of lateral LR in univariate and multivariate analyses. As a result, we studied the inter-observer correlation for metastatic characteristics of LNs on MRI. Among them, size was the most reliable variable. The cutoff value of clinically suspicious nodes was suggested as an SAD of $\geq 5 \mathrm{~mm}$ based on the ROC analysis for the size (pretreatment LLN size had the largest value of area under curve; data not shown). In the present study, patients without clinically suspected LLNs resulted in LLN recurrence in only 7 of 747 patients $(0.9 \%)$, which might have been under diagnosed, but the recurrence rate was within the acceptable range for lateral LR. Usually, the cutoff value of clinically suspicious nodes was suggested as an SAD of $\geq 5-7 \mathrm{~mm}$ or $\geq 7-8$ $\mathrm{mm}$ in the long axis diameter $[1,24-26,32,33,35]$. In the present study, 171 of 918 patients (18.6\%) had clinically suspicious LLNs, which were consistent with other reports (14\%-29.9\%) despite the studies' varied cutoff values for clinically suspicious LLNs [1, $24,25,35]$. In addition, previous studies have reported a wide range of response rates after nCRT (35.5\%-82\%), which was based on the different cutoff values of clinically suspicious nodes $[1,22,25,35]$. In the present study, the response rate was around $60 \%$, which is comparable to the results of other studies. To avoid overtreatment, it is important to determine the cutoff value of the LLN size; however, the issue lies on whether the size will be based on pretreatment MRI or restaging MRI, or both.

\section{Morbidity; is lateral pelvic lymph node dissection a really unsafe procedure? \\ Intraoperative events}

The JCOG0212 trial reported that the TME with LPLD group had a longer mean operation time (360 minutes vs. 254 minutes, $\mathrm{P}<0.0001)$ and greater mean blood loss $(576 \mathrm{~mL}$ vs. $337 \mathrm{~mL}, \mathrm{P}<$ $0.0001)$ than those of the TME-alone group [15].

To date, comparative studies between TME with LPLD and TME alone in patients with enlarged LLNs who underwent nCRT are lacking. In one small-sample RCT (22 patients in the no LPLD group, 23 patients in the LPLD group) of rectal cancer patient after nCRT, the results showed that the operation time was not different between the 2 groups, but blood loss was greater in the LPLD group [36].

Other retrospective noncomparative studies for rectal cancer patients treated with nCRT and LPLD reported long operation times (405-596 minutes) and large amounts of blood loss (715$754.5 \mathrm{~mL}$ ) [37, 38]. However, Konishi et al. [39] reported that laparoscopic LPLD resulted in a small amount of blood loss (mean, $25 \mathrm{~mL}$; range, 5-1,190 $\mathrm{mL}$ ) and shorter operation time (mean, 413 minutes; range, 277-596 minutes). Park et al. [40, 41] also reported better results for minimally invasive LPLD surgery, with a 
mean total operative time of 321.9 minutes (range, 220-510 minutes), mean robotic extended pelvic node dissection time of 38 minutes (range, 20-51 minutes), and mean operative blood loss of $188 \pm 104 \mathrm{~mL}$ (range, 50-370 mL) [40, 41].

The present study's results were similar to previous studies that reported on a minimally invasive approach. Compared to the TME-alone group, the TME with LPLD group had a longer operation time (330 minutes vs. 220 minutes, $\mathrm{P}<0.001)$ and lesser blood loss ( $100 \mathrm{~mL}$ vs. $200 \mathrm{~mL}, \mathrm{P}<0.05)$. The longer operation time and reduced blood loss of patients who underwent LPLD in the present study might be explained by more use of the laparoscopic approach, which requires a bloodless operation field and relatively low rate of bilateral LPLD.

\section{Postoperative morbidity}

The JCOG0212 trial showed similar Clavien-Dindo III and IV postoperative complications between the LPLD and TME-alone groups [15].

A previous RCT of patients treated with nCRT reported a similar complication rate between the TME with LPLD and TMEalone groups. However, compared to the LPLD group, the TMEalone group showed less urinary ( $27 \%$ vs. $65.2 \%, \mathrm{P}=0.02)$ and sexual dysfunction ( $45 \%$ vs. $92.3 \%, \mathrm{P}=0.02$ ) [36]. Recently, Akiyoshi et al. [24] reported a retrospective study that had similar rates of postoperative complications between the TME with LPLD and TME-alone groups after nCRT. Moreover, they reported very low rates of urinary retention in the LPLD ( 2 of 38 patients, 5.3\%) and TME-alone groups ( 0 of 80 patients, $0 \%$ ). Other reports also showed that LPLD did not increase the risk of postoperative complications $[37,38]$. The present study also showed similar incidence of Clavien-Dindo III and IV complications (3.9\% vs. 5.8\%) and urinary retention (13.7 vs. $10.1 \%, \mathrm{P}=0.484$ ) between the 2 groups even after nCRT, which is consistent with the findings of previous reports. In summary, the prolonged operation time with LPLD after nCRT may not necessarily lead to increased postoperative complications such as urinary retention.

What is the probability of pathologically proven metastasis in clinically suspicious lateral lymph nodes on pretreatment magnetic resonance imaging?

In the present study, LLN metastasis occurred in $34.3 \%$ of patients with enlarged LLNs with SADs of $\geq 5 \mathrm{~mm}$ on pretreatment MRI. This is comparable to other studies, which reported rates that ranged from $23.3 \%$ to $65.8 \%[1,24,32,35,38]$. Of these studies, that of Kim et al. [35] regarding patients with the same cutoff value of clinically suspicious LLNs reported a similar metastasis rate $(37.7 \%)$ as the present study.

While some studies have reported that shrunken LLNs with SADs of $<4$ or $5 \mathrm{~mm}$ after CRT have no LLN metastasis [34, 42, 43], other studies have confirmed metastasis in $9.1 \%$ to $20 \%$ of responsive nodes, including the present study (13.1\%) [1,35, 44]. On the other hand, a high rate of LLN metastasis was reported in
$61.1 \%$ to $75 \%$ of patients with persistent LLNs [42-44]. Our rate of $51.3 \%$ metastasis in persistent LLNs is comparable with those findings.

Interestingly, 13 out of 25 patients with metastatic LLNs (52.0\%) in one study [24], and 8 out of 20 patients with metastatic LLNs (40.0\%) in another study [35], had no mesorectal LN metastasis. In the present study, 10 out of 24 patients with metastatic LLNs (41.7\%) had no mesorectal LN metastasis; 9 of those were in the persistent LLN group, and 4 of the 9 patients are currently alive without disease. LR and DR were found in 1 and 4 patients, respectively.

Kanemitsu et al. [45] reported that LLN metastasis was observed in $19.1 \%$ of patients in the National Cancer Center and $15.8 \%$ of patients in Aichi Cancer Center. In addition, 23.1\% of National Cancer Center patients and 21\% of Aichi Cancer Center patients had no mesorectal lymph node metastasis.

Can lateral pelvic lymph node dissection reduce the risk of lateral local recurrence or local recurrence in patients with enlarged nodes before neoadjuvant chemoradiotherapy? Western surgeons have found that nCRT might not be sufficient to prevent LLN recurrence, which resulted in an interest in LPLD to control locoregional recurrence [23]. In addition, Akiyoshi et al. [46] revealed that lateral nodal disease could be regarded as a regional disease with a similar prognosis as an $\mathrm{N} 2$ disease. Thus, selective LPLD might play a role in the control of LR, which may possibly increase survival in patients with enlarged LLNs [23].

In the JCOG0212 trial, LPLD reduced the rates of LR and lateral LR from $13 \%$ to $7 \%$ and from $7.1 \%$ ( 25 of 350 ) to $2.0 \%$ (7 of 351 ), respectively, demonstrating that LPLD could not completely prevent lateral LR; in these cases, $\mathrm{nCRT}$ might be necessary [16]. Most studies, including the present study, have reported excellent results with LPLD after nCRT, which showed no lateral pelvic recurrence and an excellent $\mathrm{LR}$ rate $(0 \%-3.4 \%)[1,24,36,43]$.

An international multicenter pooled analysis by Lateral Node Study Consortium reported that LPLD after n(C)RT reduced the rate of LR and lateral LR from $25.6 \%$ to $5.7 \%$ and $19.56 \%$ to $5.7 \%$, respectively, in patients with LLNs with SADs $\geq 7 \mathrm{~mm}$, similar to our results. An important caveat of this study was that those who underwent LLN sampling only and not regional lymphadenectomy (LPLD) showed a very high rate of lateral recurrence (around 50\%) [25]. Another recent multicenter comparative study also showed that LPLD was an independent risk factor for LR on multivariable analysis [47]. Our study showed consistent findings with previous reports that LPLD reduced the 5-year LR rate from $27.9 \%$ to $4.6 \%$. Undoubtedly, LPLD is beneficial in preventing lateral pelvic recurrence.

\section{Can lateral pelvic lymph node dissection increase recurrence-free survival or overall survival in patients with enlarged nodes before neoadjuvant chemoradiotherapy? \\ Since locoregional control is the primary goal of surgery, most}


Western surgeons believe that LPLD does not seem to decrease the rate of distant metastasis, just as RT is not effective in reducing distant recurrence. The JCOG0212 trial showed a similar 5-year RFS and OS in the TME with LPLD and TME-alone groups [16].

On the other hand, the CSS was higher in the Japanese LPLD group than both the Dutch nonirradiated TME group and Dutch RT + TME group [18]. An international multicenter pooled analysis also showed a better 5-year DR and CSS in the LPLD group compared with the TME-alone group ( $13.5 \%$ vs. $30.8 \%, \mathrm{P}=0.028$; $94.1 \%$ vs. $79.4 \%, \mathrm{P}=0.032$, respectively) [25]. In our present study, a quite similar feature to the international pooled analysis was observed regarding the 5-year DR between the TME only and LPLD groups (34.1\% vs. $15.0 \%, \mathrm{P}=0.005)$. The 5 -year CSS and OS were increased after LPLD; however, statistical significance was not reached $(78.7 \%$ vs. $89.3 \%, \mathrm{P}=0.068 ; 76.2 \%$ vs. $86.5 \%, \mathrm{P}=0.094$, respectively). The present study also showed a significantly better RFS in the LPLD group (59.6\% vs. $78.2 \%, P=0.008)$. In summary, LPLD seems to increase RFS but does not necessarily resulted in a better CSS or OS. Further studies with larger numbers of patients would be necessary to prove the survival benefit of LPLD.

\section{Subgroup analysis of the responsive and persistent groups}

Given the complications accompanying LPLD and relatively low rate of LLN metastasis, it is important to select patients from whom you can expect therapeutic benefit from LPLD [7].

Even though LLNs with SADs of $\geq 5$ to $7 \mathrm{~mm}$ on MRI is an important predictor of LLN metastasis $[25,26,35]$, there have been debates on whether the cutoff value of the LLN size should be determined by the pretreatment MRI or the restaging MRI.

Ogura et al. $[25,34]$ reported that an SAD of $\leq 4 \mathrm{~mm}$ after nCRT did not show any lateral LR at 3 years; thus they suggest an $\mathrm{SAD}$ of $\geq 4 \mathrm{~mm}$ after nCRT as a safe cutoff value for LPLD. Based on these findings, LPLD can be avoided around $30 \%$ of the time. However, in the same study, a 20\% lateral LR was found in patients with LLN of $\leq 4 \mathrm{~mm}$ after 5 years [34]. Yeo et al. [48] reported that delayed LR (13.2\%) after 5 years was observed more frequently in patients treated with nCRT than patients treated with postoperative CRT (4.3\%), and the 10-year follow-up of the COREAN trial also reported that delayed recurrence after 5 years was observed in $17 \%$ of all LRs and $8 \%$ of all DRs [49]. Theoretically, the smaller the LLN size, the greater the probability of delayed recurrence; hence, the cutoff value should be set with the lateral LR rate at 5 years.

On the other hand, previous studies have confirmed metastasis in $9.1 \%$ to $20 \%$ of patients with responsive LLNs $[1,26,35,44]$. Moreover, a histological study with patients treated within the German Rectal Cancer Trial CAO/ARO/AIO-04 reported that micrometastases $(<0.2 \mathrm{~cm}$ ) accounted for $28.3 \%$ of all LN metastases, and $50 \%$ of metastatic nodes were LNs of $<3 \mathrm{~mm}$ after nCRT [50]. Based on these findings, LPLD should be necessary in patients with shrunken LLNs with SADs of $<4 \mathrm{~mm}$ after nCRT.

Matsuda et al. [38] reported that there was no LR after LPLD in good responders and a high rate (42.9\%) of LR in poor responders, which suggested that selective LPLD is effective only in good responders. Kim et al. [35] also did not report any LR in good responders, but $21.7 \%$ of patients ( 5 of 23 ) developed LR in the persistent LLN group, which was a similar proportion $(22.6 \%, 6$ of 31 ) to that of the no LPLD group. Thus, they also insisted that LPLD was more beneficial in the good response group. However, considering that 6 of 31 good responders (19.4\%) who did not undergo LPLD had recurrence in the lateral pelvic sidewall, only 2 of 23 poor responders (8.7\%) experienced pelvic sidewall recurrences, which revealed an excellent therapeutic benefit of LPLD even in poor responders. Many studies did not report lateral LR after LPLD, irrespective of the shrinkage of the LLNs after nCRT $[1,24,36,43]$. Therefore, our institutional policy for LPLD has not changed, and the cutoff value for LPLD remains $\geq 5 \mathrm{~mm}$ on pretreatment MRI irrespective of the response to nCRT.

Generally, the cutoff value for LPLD is defined as LLNs with SADs of $\geq 5$ to $7 \mathrm{~mm}$ on pretreatment MRI or an SAD of 4 to 5 $\mathrm{mm}$ on restaging MRI. According to the size and response of the LLNs, a clinical algorithm has been suggested for reference based on the data from the Lateral Node Study Consortium [3, 25, 51]. Further research is needed to define the optimal cutoff value of LLNs for LPLD, considering the sensitivity and specificity of clinically suspicious LLNs on MRI. The number of false-negative cases can be decreased by setting a small cutoff value and accepting an increase in the number of false-positive cases [32]. In addition, surgical skills and a multidisciplinary team approach for LPLD should also be important factors in determining the cutoff value of LLNs for LPLD.

\section{Limitations}

Our study has several limitations. First, this is a single center, retrospective, and observational study. Therefore, we could not avoid selection bias and could not generalize the results of this study. Second, we compared the outcomes between the patients who received LPLD and those who did not after nCRT using historical controls. To evaluate treatment-related bias according to the treatment period, we compared the oncological outcomes of patients without enlarged LLNs who underwent TME alone according to the 2 periods. The results showed similar oncological outcomes between treatment periods; thus, we could assume that the difference of the treatment effects according to the period was minimal [26]. Third, the recent introduction of LPLD might have had an insufficient observation period compared to that of the historical controls. Thus, we adjusted the follow-up periods of both groups to be similar. Considering that lateral LR did not occur in the LPLD group in this study and delayed recurrence after the 5-year follow-up was $7 \%$ to $10 \%$ of all recurrences $[48,49]$, a possible number of events regarding the delayed recurrence would be minimal. Taken together, the oncological outcomes of the TMEalone group (period I) are more likely to be better than those of the LPLD group (period II). Lastly, the sample size of this study is 
not sufficient, which resulted in the lack of statistical power to interpret the data. Further studies with larger numbers of patients would be necessary to confirm our results.

\section{Future perspectives}

Application of pelvic autonomic nerve-sparing techniques and recent advances in instruments and magnified imaging systems in minimally invasive approaches have enabled surgeons to safely perform LPLD. In addition, many video materials are also helpful for inexperienced surgeons to learn LPLD. However, a recent survey on lateral pelvic LNs in rectal cancer across members of the Colorectal Surgical Society of Australia and New Zealand showed more than half $(60 \%)$ of surgeons did not have any experience with LPLD during their training, and only $21 \%$ performed more than 1 to 2 cases of LPLD per year. In addition, only $4 \%$ of surgeons performed LPLD more than 10 times per year [52].

A prospective RCT for LPLD after nCRT was conducted in China (ClinicalTrials.gov identifier: NCT02614157) that included 512 patients who had LLN of $>5$ and $<10 \mathrm{~mm}$ in SAD to show an $8 \%$ difference LR. Unfortunately, this study was terminated without any results [53].

Recently, an international prospect registration study (Lateral Nodal Recurrence in Rectal Cancer, LaNoReC; ClinicalTrials.gov identifier: NCT04486131) is currently being conducted by Kusters et al., VU University Medical Center, Netherlands to increase national awareness for enlarged lateral nodes and their role in LRs. The main question of this study is whether selective LPLD after nCRT performed in a dedicated center can reduce the lateral LR rate in patients with LLN of $\geq 7 \mathrm{~mm}$ to below $6 \%$.

In Korea, we started the "Lateral Node Study Group" in 2017. Since then, we have organized 3 cadaver workshops and several conferences with Japanese surgeons. We have also developed a standard technique for LPLD and a web-based registration system for a prospective registration study. This study would provide further insights into lateral pelvic nodal disease and help clinical decision making.

Recent advanced technologies, such as indocyanine green or near-infrared fluorescence imaging, have been studied for intraoperative LN mapping in LPLD to improve the LN yield and avoid incomplete LPLD [54]. Sentinel LN techniques, which have demonstrated disappointing results in determining LN metastasis in colorectal cancer, could be revisited during transanal TME [55]. A transanal approach to the lateral space could offer new ideas for sentinel LN techniques to avoid unnecessary systematic lateral lymphadenectomy.

\section{Conclusion}

This study included a relatively larger number of patients compared to other single-center studies. Our results suggest that LPLD is a safe and feasible procedure. Selective LPLD might improve the rate of LR and relapse-free survival in patients who have LLNs with SADs of $\geq 5 \mathrm{~mm}$ on pretreatment MRI. Considering that lat- eral nodal disease is relatively uncommon, a multicenter largescale study such as a prospective registration study is necessary.

\section{CONFLICTS OF INTEREST}

No potential conflicts of interest relevant to this article were reported.

\section{ACKNOWLEDGMENTS}

The authors are grateful to Eun Young Park and Kiho You, M.D. for their contributions to the statistical analysis of the data.

\section{SUPPLEMENTARY MATERIALS}

Supplementary materials for this study are presented online (available at https://doi.org/10.3393/ac.2021.00913.0130).

\section{REFERENCES}

1. Ishihara S, Kawai K, Tanaka T, Kiyomatsu T, Hata K, Nozawa H, et al. Oncological outcomes of lateral pelvic lymph node metastasis in rectal cancer treated with preoperative chemoradiotherapy. Dis Colon Rectum 2017;60:469-76.

2. Enker WE, Laffer UT, Block GE. Enhanced survival of patients with colon and rectal cancer is based upon wide anatomic resection. Ann Surg 1979;190:350-60.

3. Williamson JS, Quyn AJ, Sagar PM. Rectal cancer lateral pelvic sidewall lymph nodes: a review of controversies and management. Br J Surg 2020;107:1562-9.

4. Kim S. Distribution of lymph nodes in stage III patients with mid and low rectal cancer: preliminary study. Ann Coloproctol 2018; 34:42-6.

5. Kuru M. Cancer of the rectum. J Jpn Surg Soc 1940:832-77.

6. Takahashi T, Ueno M, Azekura K, Ohta H. Lateral node dissection and total mesorectal excision for rectal cancer. Dis Colon Rectum 2000;43(10 Suppl):S59-68.

7. Hashiguchi Y, Muro K, Saito Y, Ito Y, Ajioka Y, Hamaguchi T, et al. Japanese Society for Cancer of the Colon and Rectum (JSCCR) guidelines 2019 for the treatment of colorectal cancer. Int J Clin Oncol 2020;25:1-42.

8. Tiefenbacher U, Wenz F. Preoperative radiotherapy combined with total mesorectal excision for resectable rectal cancer. Strahlenther Onkol 2001;177:682-4.

9. Sauer R, Becker H, Hohenberger W, Rödel C, Wittekind C, Fietkau $\mathrm{R}$, et al. Preoperative versus postoperative chemoradiotherapy for rectal cancer. N Engl J Med 2004;351:1731-40.

10. Kim MJ, Jeong SY, Park JW, Ryoo SB, Cho SS, Lee KY, et al. Oncologic outcomes in patients who undergo neoadjuvant chemoradiotherapy and total mesorectal excision for locally advanced rectal cancer: a 14-year experience in a single institution. Ann Coloproctol 2019;35:83-93. 
11. Yoo RN, Kim HJ. Organ preservation strategies after neoadjuvant chemoradiotherapy for locally advanced rectal cancer. Ann Coloproctol 2019;35:53-64.

12. Georgiou P, Tan E, Gouvas N, Antoniou A, Brown G, Nicholls RJ, et al. Extended lymphadenectomy versus conventional surgery for rectal cancer: a meta-analysis. Lancet Oncol 2009;10:1053-62.

13. Emile SH, Elfeki H, Shalaby M, Sakr A, Kim NK. Outcome of lateral pelvic lymph node dissection with total mesorectal excision in treatment of rectal cancer: a systematic review and meta-analysis. Surgery 2021;169:1005-15.

14. Kong JCH, Chang GJ. Apples and oranges: the evidence regarding lateral pelvic lymph node dissection for rectal cancer. Surgery 2021;169:1003-4.

15. Fujita S, Akasu T, Mizusawa J, Saito N, Kinugasa Y, Kanemitsu Y, et al. Postoperative morbidity and mortality after mesorectal excision with and without lateral lymph node dissection for clinical stage II or stage III lower rectal cancer (JCOG0212): results from a multicentre, randomised controlled, non-inferiority trial. Lancet Oncol 2012;13:616-21.

16. Fujita S, Mizusawa J, Kanemitsu Y, Ito M, Kinugasa Y, Komori K, et al. Mesorectal excision with or without lateral lymph node dissection for clinical stage II/III lower rectal cancer (JCOG0212): a multicenter, randomized controlled, noninferiority trial. Ann Surg 2017;266:201-7.

17. Saito S, Fujita S, Mizusawa J, Kanemitsu Y, Saito N, Kinugasa Y, et al. Male sexual dysfunction after rectal cancer surgery: results of a randomized trial comparing mesorectal excision with and without lateral lymph node dissection for patients with lower rectal cancer. Japan Clinical Oncology Group Study JCOG0212. Eur J Surg Oncol 2016;42:1851-8.

18. Kusters M, Beets GL, van de Velde CJ, Beets-Tan RG, Marijnen $\mathrm{CA}$, Rutten HJ, et al. A comparison between the treatment of low rectal cancer in Japan and the Netherlands, focusing on the patterns of local recurrence. Ann Surg 2009;249:229-35.

19. Kim JC, Takahashi K, Yu CS, Kim HC, Kim TW, Ryu MH, et al. Comparative outcome between chemoradiotherapy and lateral pelvic lymph node dissection following total mesorectal excision in rectal cancer. Ann Surg 2007;246:754-62.

20. Kim TH, Jeong SY, Choi DH, Kim DY, Jung KH, Moon SH, et al. Lateral lymph node metastasis is a major cause of locoregional recurrence in rectal cancer treated with preoperative chemoradiotherapy and curative resection. Ann Surg Oncol 2008;15:729-37.

21. Kim MJ, Kim TH, Kim DY, Kim SY, Baek JY, Chang HJ, et al. Can chemoradiation allow for omission of lateral pelvic node dissection for locally advanced rectal cancer? J Surg Oncol 2015;111: 459-64.

22. Kim MJ, Chan Park S, Kim TH, Kim DY, Kim SY, Baek JY, et al. Is lateral pelvic node dissection necessary after preoperative chemoradiotherapy for rectal cancer patients with initially suspected lateral pelvic node? Surgery 2016;160:366-76.

23. Kusters M, Slater A, Muirhead R, Hompes R, Guy RJ, Jones OM, et al. What to do with lateral nodal disease in low locally ad- vanced rectal cancer? A call for further reflection and research. Dis Colon Rectum 2017;60:577-85.

24. Akiyoshi T, Ueno M, Matsueda K, Konishi T, Fujimoto Y, Nagayama S, et al. Selective lateral pelvic lymph node dissection in patients with advanced low rectal cancer treated with preoperative chemoradiotherapy based on pretreatment imaging. Ann Surg Oncol 2014;21:189-96.

25. Ogura A, Konishi T, Cunningham C, Garcia-Aguilar J, Iversen H, Toda S, et al. Neoadjuvant (chemo)radiotherapy with total mesorectal excision only is not sufficient to prevent lateral local recurrence in enlarged nodes: results of the multicenter lateral node study of patients with low cT3/4 rectal cancer. J Clin Oncol 2019; 37:33-43.

26. Kim MJ, Chang GJ, Lim HK, Song MK, Park SC, Sohn DK, et al. Oncological impact of lateral lymph node dissection after preoperative chemoradiotherapy in patients with rectal cancer. Ann Surg Oncol 2020;27:3525-33.

27. Ueno M, Oya M, Azekura K, Yamaguchi T, Muto T. Incidence and prognostic significance of lateral lymph node metastasis in patients with advanced low rectal cancer. Br J Surg 2005;92:756-63.

28. Benson AB, Venook AP, Al-Hawary MM, Cederquist L, Chen YJ, Ciombor KK, et al. Rectal cancer, version 2.2018, NCCN Clinical Practice Guidelines in Oncology. J Natl Compr Canc Netw 2018; 16:874-901.

29. Beets-Tan RG, Beets GL. Rectal cancer: review with emphasis on MR imaging. Radiology 2004;232:335-46.

30. Kim JH, Beets GL, Kim MJ, Kessels AG, Beets-Tan RG. High-resolution MR imaging for nodal staging in rectal cancer: are there any criteria in addition to the size? Eur J Radiol 2004;52:78-83.

31. Seo N, Kim H, Cho MS, Lim JS. Response Assessment with MRI after chemoradiotherapy in rectal cancer: current evidences. Korean J Radiol 2019;20:1003-18.

32. Ogawa S, Hida J, Ike H, Kinugasa T, Ota M, Shinto E, et al. Selection of lymph node-positive cases based on perirectal and lateral pelvic lymph nodes using magnetic resonance imaging: study of the Japanese Society for Cancer of the Colon and Rectum. Ann Surg Oncol 2016;23:1187-94.

33. Ogawa S, Hida J, Ike H, Kinugasa T, Ota M, Shinto E, et al. The important risk factor for lateral pelvic lymph node metastasis of lower rectal cancer is node-positive status on magnetic resonance imaging: study of the Lymph Node Committee of Japanese Society for Cancer of the Colon and Rectum. Int J Colorectal Dis 2016;31:1719-28.

34. Ogura A, Konishi T, Beets GL, Cunningham C, Garcia-Aguilar J, Iversen $\mathrm{H}$, et al. Lateral nodal features on restaging magnetic resonance imaging associated with lateral local recurrence in low rectal cancer after neoadjuvant chemoradiotherapy or radiotherapy. JAMA Surg 2019;154:e192172.

35. Kim HJ, Choi GS, Park JS, Park SY, Cho SH, Lee SJ, et al. Optimal treatment strategies for clinically suspicious lateral pelvic lymph node metastasis in rectal cancer. Oncotarget 2017;8:100724-33.

36. Nagawa H, Muto T, Sunouchi K, Higuchi Y, Tsurita G, Watanabe 
T, et al. Randomized, controlled trial of lateral node dissection vs. nerve-preserving resection in patients with rectal cancer after preoperative radiotherapy. Dis Colon Rectum 2001;44:1274-80.

37. Otowa Y, Yamashita K, Kanemitsu K, Sumi Y, Yamamoto M, Kanaji S, et al. Treating patients with advanced rectal cancer and lateral pelvic lymph nodes with preoperative chemoradiotherapy based on pretreatment imaging. Onco Targets Ther 2015;8:3169-73.

38. Matsuda T, Sumi Y, Yamashita K, Hasegawa H, Yamamoto M, Matsuda Y, et al. Outcomes and prognostic factors of selective lateral pelvic lymph node dissection with preoperative chemoradiotherapy for locally advanced rectal cancer. Int J Colorectal Dis 2018;33:367-74.

39. Konishi T, Kuroyanagi H, Oya M, Ueno M, Fujimoto Y, Akiyoshi $\mathrm{T}$, et al. Multimedia article. Lateral lymph node dissection with preoperative chemoradiation for locally advanced lower rectal cancer through a laparoscopic approach. Surg Endosc 2011;25: 2358-9.

40. Park JS, Choi GS, Lim KH, Jang YS, Kim HJ, Park SY, et al. Laparoscopic extended lateral pelvic node dissection following total mesorectal excision for advanced rectal cancer: initial clinical experience. Surg Endosc 2011;25:3322-9.

41. Park JA, Choi GS, Park JS, Park SY. Initial clinical experience with robotic lateral pelvic lymph node dissection for advanced rectal cancer. J Korean Soc Coloproctol 2012;28:265-70.

42. Oh HK, Kang SB, Lee SM, Lee SY, Ihn MH, Kim DW, et al. Neoadjuvant chemoradiotherapy affects the indications for lateral pelvic node dissection in mid/low rectal cancer with clinically suspected lateral node involvement: a multicenter retrospective cohort study. Ann Surg Oncol 2014;21:2280-7.

43. Malakorn S, Yang Y, Bednarski BK, Kaur H, You YN, Holliday EB, et al. Who should get lateral pelvic lymph node dissection after neoadjuvant chemoradiation? Dis Colon Rectum 2019;62:115866.

44. Akiyoshi T, Matsueda K, Hiratsuka M, Unno T, Nagata J, Nagasaki $\mathrm{T}$, et al. Indications for lateral pelvic lymph node dissection based on magnetic resonance imaging before and after preoperative chemoradiotherapy in patients with advanced low-rectal cancer. Ann Surg Oncol 2015;22 Suppl 3:S614-20.

45. Kanemitsu Y, Komori K, Shida D, Ochiai H, Tsukamoto S, Kinoshita T, et al. Potential impact of lateral lymph node dissection (LLND) for low rectal cancer on prognoses and local control: a comparison of 2 high-volume centers in Japan that employ different policies concerning LLND. Surgery 2017;162:303-14.
46. Akiyoshi T, Watanabe T, Miyata S, Kotake K, Muto T, Sugihara K, et al. Results of a Japanese nationwide multi-institutional study on lateral pelvic lymph node metastasis in low rectal cancer: is it regional or distant disease? Ann Surg 2012;255:1129-34.

47. Kroon HM, Malakorn S, Dudi-Venkata NN, Bedrikovetski S, Liu J, Kenyon-Smith T, et al. Local recurrences in western low rectal cancer patients treated with or without lateral lymph node dissection after neoadjuvant (chemo)radiotherapy: an international multi-centre comparative study. Eur J Surg Oncol 2021;47:2441-9.

48. Yeo SG, Kim MJ, Kim DY, Chang HJ, Kim MJ, Baek JY, et al. Patterns of failure in patients with locally advanced rectal cancer receiving pre-operative or post-operative chemoradiotherapy. Radiat Oncol 2013;8:114.

49. Park JW, Kang SB, Hao J, Lim SB, Choi HS, Kim DW, et al. Open versus laparoscopic surgery for mid or low rectal cancer after neoadjuvant chemoradiotherapy (COREAN trial): 10-year follow-up of an open-label, non-inferiority, randomised controlled trial. Lancet Gastroenterol Hepatol 2021;6:569-77.

50. Sprenger T, Rothe H, Homayounfar K, Beissbarth T, Ghadimi $\mathrm{BM}$, Becker $\mathrm{H}$, et al. Preoperative chemoradiotherapy does not necessarily reduce lymph node retrieval in rectal cancer specimens: results from a prospective evaluation with extensive pathological work-up. J Gastrointest Surg 2010;14:96-103.

51. Fung D, Lee P. Update on indications for lateral lymph node dissection in the management of lower rectal cancer. ANZ J Surg 2020;90:1265-9.

52. Cribb B, Kong J, McCormick J, Warrier S, Heriot A. Lateral pelvic lymph node dissection for rectal cancer: unfinished business? ANZ J Surg 2020;90:1228-9.

53. Wei M, Wu Q, Fan C, Li Y, Chen X, Zhou Z, et al. Lateral pelvic lymph node dissection after neoadjuvant chemo-radiation for preoperative enlarged lateral nodes in advanced low rectal cancer: study protocol for a randomized controlled trial. Trials 2016;17: 561.

54. Emile SH, Elfeki H, Shalaby M, Sakr A, Sileri P, Laurberg S, et al. Sensitivity and specificity of indocyanine green near-infrared fluorescence imaging in detection of metastatic lymph nodes in colorectal cancer: systematic review and meta-analysis. J Surg Oncol 2017;116:730-40.

55. van der Pas MH, Meijer S, Hoekstra OS, Riphagen II, de Vet HC, Knol DL, et al. Sentinel-lymph-node procedure in colon and rectal cancer: a systematic review and meta-analysis. Lancet Oncol 2011;12:540-50. 